\title{
On the global trends and spread of the COVID-19 outbreak: preliminary assessment of the potential relation between location-specific temperature and UV index
}

\author{
Sachin S. Gunthe ${ }^{1} \cdot$ Basudev Swain ${ }^{1} \cdot$ Satya S. Patra ${ }^{2} \cdot$ Aneesh Amte $^{3}$ \\ Received: 20 March 2020 / Accepted: 1 April 2020 / Published online: 24 April 2020 \\ (C) Springer-Verlag GmbH Germany, part of Springer Nature 2020
}

\begin{abstract}
The novel coronavirus, since its first outbreak in December, has, up till now, affected approximately 114,542 people across 115 countries. Many international agencies are devoting efforts to enhance the understanding of the evolving COVID-19 outbreak on an international level, its influences, and preparedness. At present, COVID-19 appears to affect individuals through person-toperson means, like other commonly found cold or influenza viruses. It is widely known and acknowledged that viruses causing influenza peak during cold temperatures and gradually subside in the warmer temperature, owing to their seasonality. Thus, COVID-19, due to its regular flu-like symptoms, is also expected to show similar seasonality and subside as the global temperatures rise in the northern hemisphere with the onset of spring. Despite these speculations, however, the systematic analysis in the global perspective of the relation between COVID-19 spread and meteorological parameters is unavailable. Here, by analyzing the region- and city-specific affected global data and corresponding meteorological parameters, we show that there is an optimum range of temperature and UV index strongly affecting the spread and survival of the virus, whereas precipitation, relative humidity, cloud cover, etc. have no effect on the virus. Unavailability of pharmaceutical interventions would require greater preparedness and alert for the effective control of COVID-19. Under these conditions, the information provided here could be very helpful for the global community struggling to fight this global crisis. It is, however, important to note that the information presented here clearly lacks any physiological evidences, which may merit further investigation. Thus, any attempt for management, implementation, and evaluation strategies responding to the crisis arising due to the COVID-19 outbreak must not consider the evaluation presented here as the foremost factor.
\end{abstract}

Keywords Coronavirus · COVID-19 $\cdot$ Temperature $\cdot$ UV index $\cdot$ Non-physiological reaction

\section{Introduction}

As of 10th March 2020, 114,814 confirmed cases of novel coronavirus (also called COVID-19) infection have been reported worldwide since its emergence in mid-December $(\mathrm{Du}$

Sachin S. Gunthe

s.gunthe@iitm.ac.in

1 EWRE Division, Department of Civil Engineering, Indian Institute of Technology Madras, Chennai 600 036, India

2 Transportation Engineering Division, Department of Civil Engineering, Indian Institute of Technology Madras, Chennai 600 036, India

3 Aasha Endoscopy Center, Vasant Prestige, 5 Bunglow, Shahupuri, Kolhapur 416001, India
Toit 2020). Almost 70.3\% are being reported from China alone, followed by Italy (8.0\%), South Korea (6.5\%), Iran (6.2\%), France $(1.2 \%)$, Spain (1\%), Germany (1\%), the USA $(0.6 \%)$, and Japan $(0.6 \%)$, constituting more than $\sim 95 \%$ of the total reported confirmed cases. In a report released by the World Health Organization (WHO). a major goal and objective is outlined to rapidly disseminate the national (for China) and international planning on the preparedness in response to the increasing and ongoing outbreak of COVID19. The virus traces its origin for the recent outbreak in China, when three samples for bronchoalveolar lavage were collected and detailed microbiological analysis confirmed that the virus had typical features resembling the coronavirus family. COVID-19 has a transmission characteristic typical of any other cold-causing influenza virus: human-to-human. As per the report published by the WHO, the common symptoms 
include fever, dry cough, fatigue, sputum production, shortness of breath, sore throat, headache, etc., which can be recognized as normal flu symptoms.

Since the beginning of the COVID-19 outbreak, extensive efforts have been constantly devoted to improving understanding about the virus. As a result, this virus, previously unknown to many, not only garnered tremendous research attention, but also became a household name within a span of merely 90 days. The outbreak has also brought many major cities in China to a standstill and resulted in the isolation of a large number of citizens in Italy. Such a curfew-like situation can be difficult to impose and may not yield the desired results under many circumstances (Fong et al. 2020), which is evident from the steps being taken by countries to contain further spread of the virus. At the same time, another prevalent issue is considering the COVID-19 features that are similar to common influenza viruses causing colds. The most common feature of common influenza viruses is seasonality, which implies that, during colder temperatures, influenza caused by viruses increases, which then subside with the warming of air temperature. This assumption, at this moment, is not backed by any robust analysis and scientific investigations. Under the circumstances of unobtainability of any pharmaceutical relief to treat the COVID-19 virus, specific information about the non-physiological causes augmenting the survival and spread of COVID-19 can be very valuable information for policy-makers dealing with management and effective control measures. Since the onset of the COVID-19 outbreak, a lot of attention has been paid to understanding cross-species transmission (Ji et al. 2020), efforts regarding the discovery, emergence, clinical diagnostic, and genomic characteristics of COVID-19 (Phan 2020), and initial public response (Patel et al. 2020). Further, there are requirements for details related to the necessity of maintaining regular physical activity while exercising precautions (Chen et al. 2020) and, more interestingly, even developing an accurate model to forecast the novel COVID-19 outbreak from small datasets (Fong et al. 2020). The relation of COVID-19 with meteorological parameters, which are very location-specific where outbreaks have been reported, however, has not been investigated. In this study, we analyzed the number of confirmed cases up till 7 th March 2020 and investigated location-specific outbreaks with meteorological parameters, including temperature, relative humidity, UV index, and precipitation.

\section{Materials and methods}

To better understand the demographic distribution of COVID19 on the global scale, we used data that were not derived for any in situ measurements or monitoring. Instead, we captured the country-/region-dependent spread from alerts generated by the Program for Monitoring Emerging Diseases (ProMED), which is a community-based online reporting system (Yadav et al. 2020). The very fact that reports in the ProMED are fully screened by a group of expert members for its validity makes the reports reliable to be used in scientific publishing (Fisher et al. 2012). We used the key word "COVID-19" with the option enabled for subject and post; for exclusions, see also the ProMED-mail database. Thereafter, we selectively screened the number of infected cases listed for each country from 2nd February 2020, where the accumulated infected cases were already provided. As shown in Table 1, we considered the cumulative dataset for 85 locations, including the highly affected provinces in China, South Korea, Italy, Iran, France, Germany, the USA, Spain, and Japan. Further, the meteorological data for minimum, maximum, and average temperature (in ${ }^{\circ} \mathrm{C}$ ); relative humidity (in \%), UV index (an index indicating the strength of sunburn-producing UV radiation), precipitation (in $\mathrm{mm}$ ), and cloud cover were obtained from the website https://www.worldweatheronline.com (last accessed 10th March 2020, 04:30 h IST), which is dedicated to providing global weather content and has been used by previous researchers (Ibekwe and Ukonu 2019). The providers claim to cover around 3 million sites across the globe, and the data are used by many organizations. While it is difficult to independently verify the quality and accuracy of the data, random validation for a few cities with actual measurements in the past have given a strong agreement between the two datasets. To obtain the data for a specific city, we applied the search criteria by narrowing on the province/region and then querying the most affected cities in the respective province/region obtained from various other data sources. Thus, we are of the strong opinion that the meteorological data presented here are not only the true representation of the city/place with infected cases, but also give an accurate indication of the relation between the number of confirmed cases and the meteorological parameters.

Further, for the countries with more than 500 confirmed cases, we only chose cities that constituted more than $70 \%$ of the total confirmed cases for that respective country. Thus, we scrutinized a total of 107,351 confirmed cases across 85 locations.

Once the data were obtained, we prepared scatter plots between the number of confirmed cases and various meteorological parameters. Further, by applying various statistical fits, the dependence of virus spread on individual meteorological parameters was investigated, as discussed below.

\section{Assessment}

Figure 1 shows the relation between the number of confirmed cases and temperature assessed for more than 10,000 cases globally, constituting $\sim 85$ individual locations. As can be seen from Fig. 1a, the data points are clearly following the log 


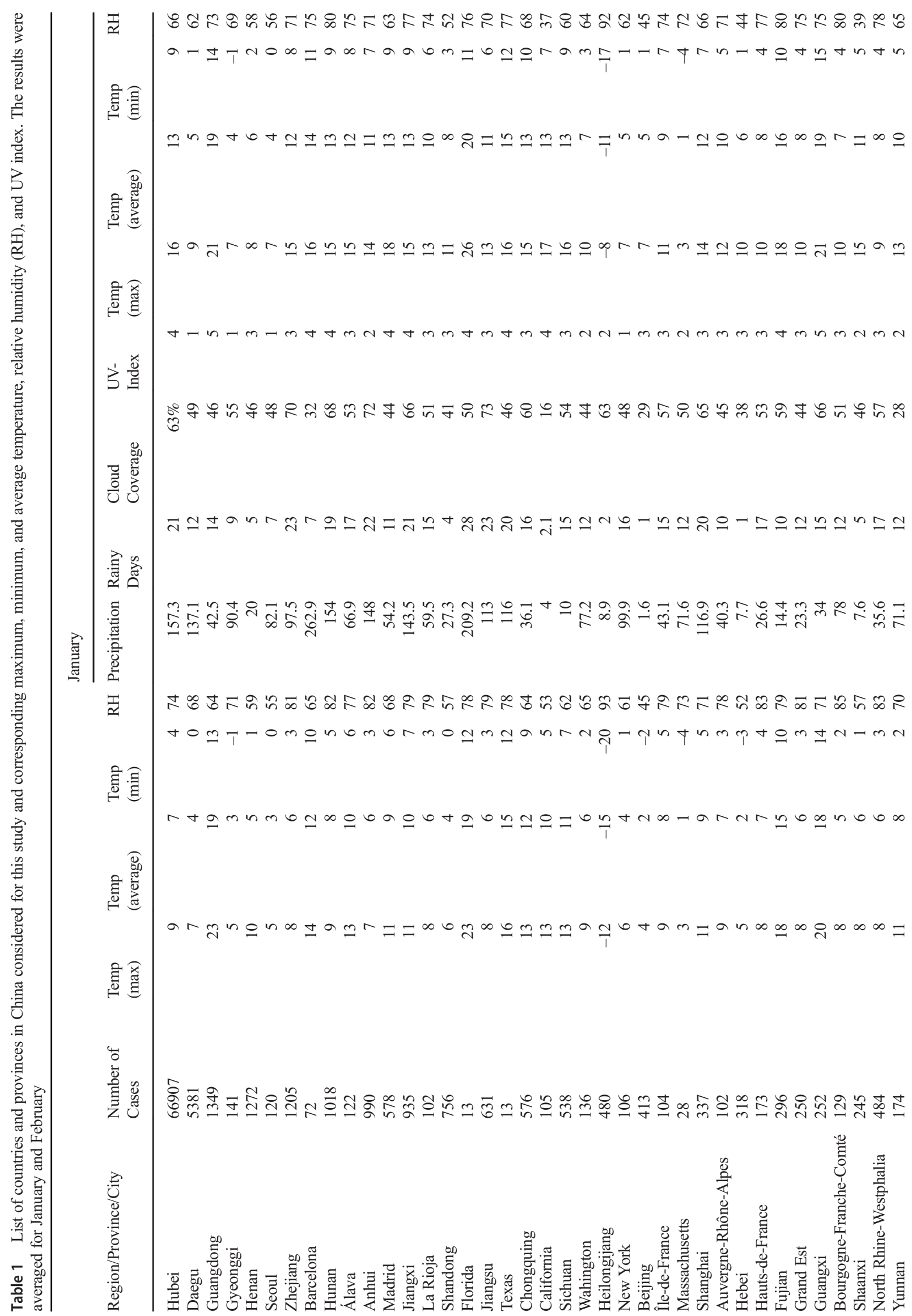




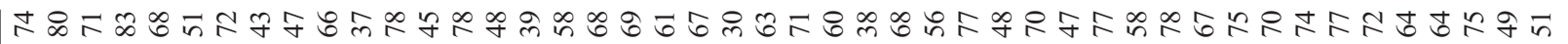
manR -

$\wedge$ ป

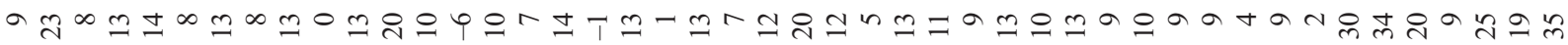

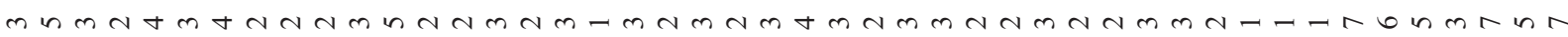

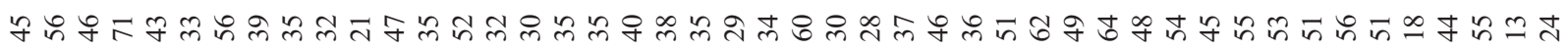

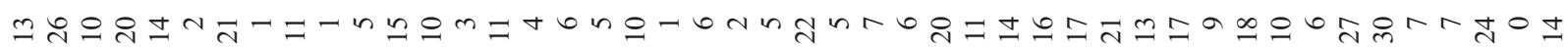

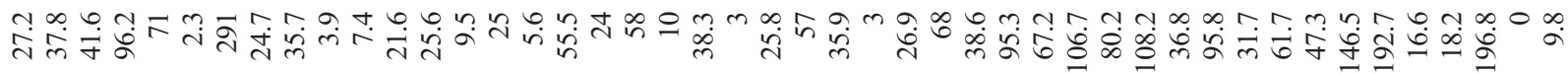

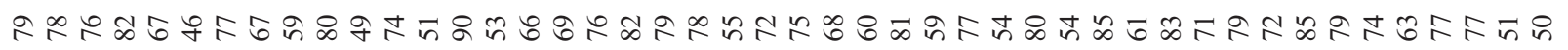
No 0 的 n ก ก

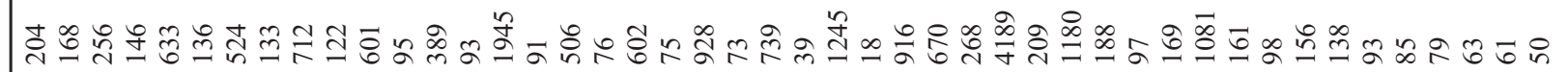




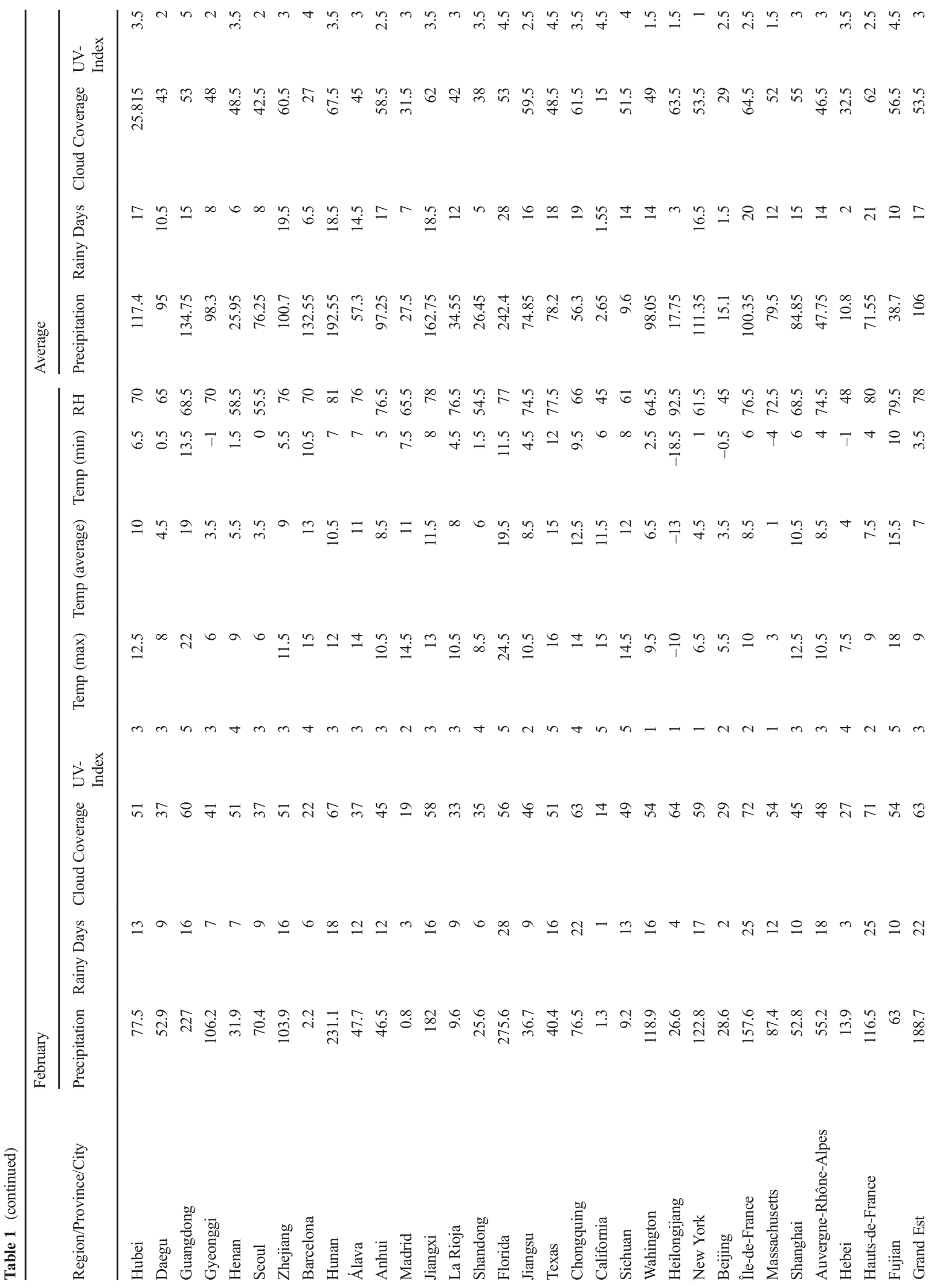




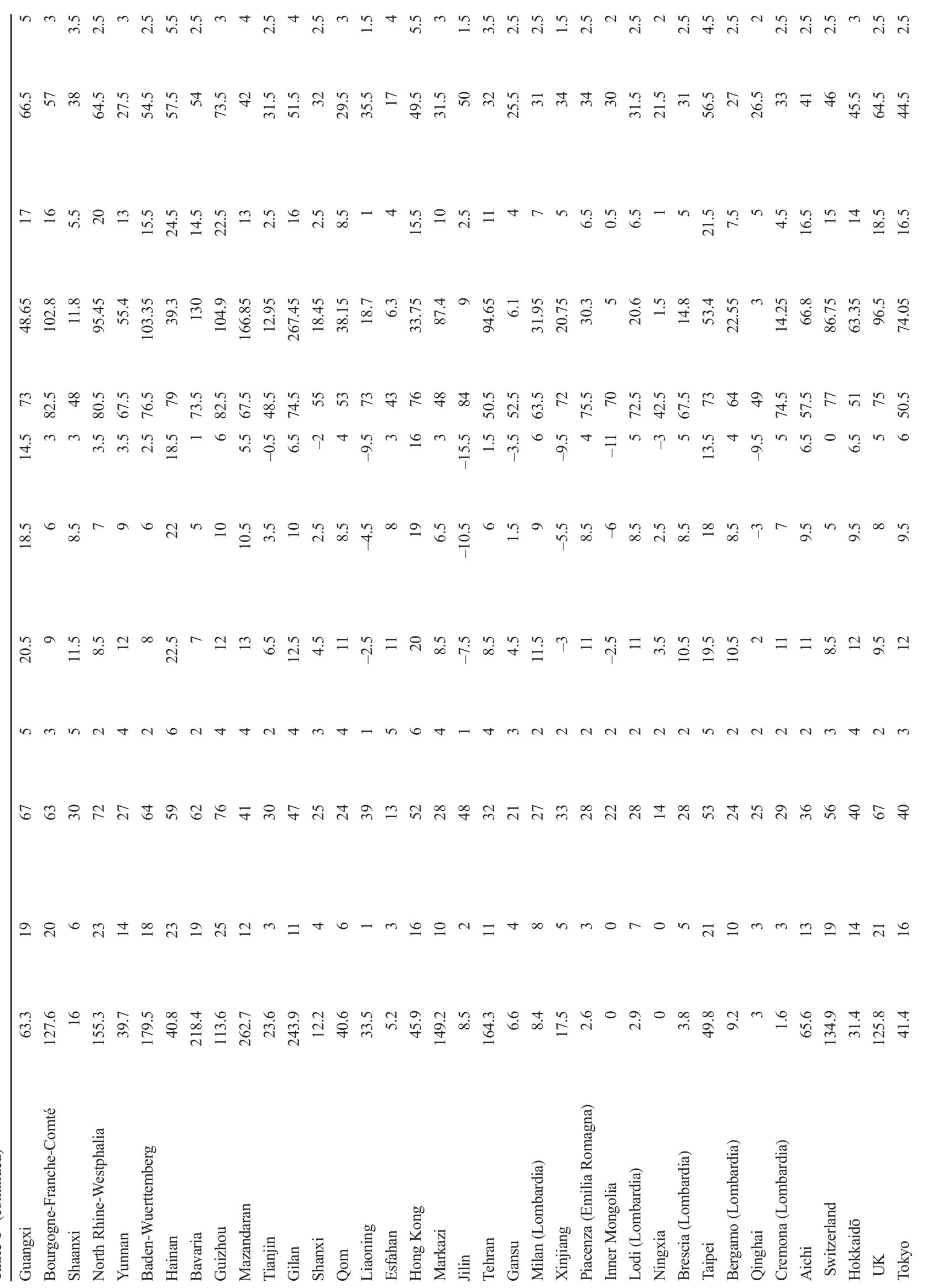




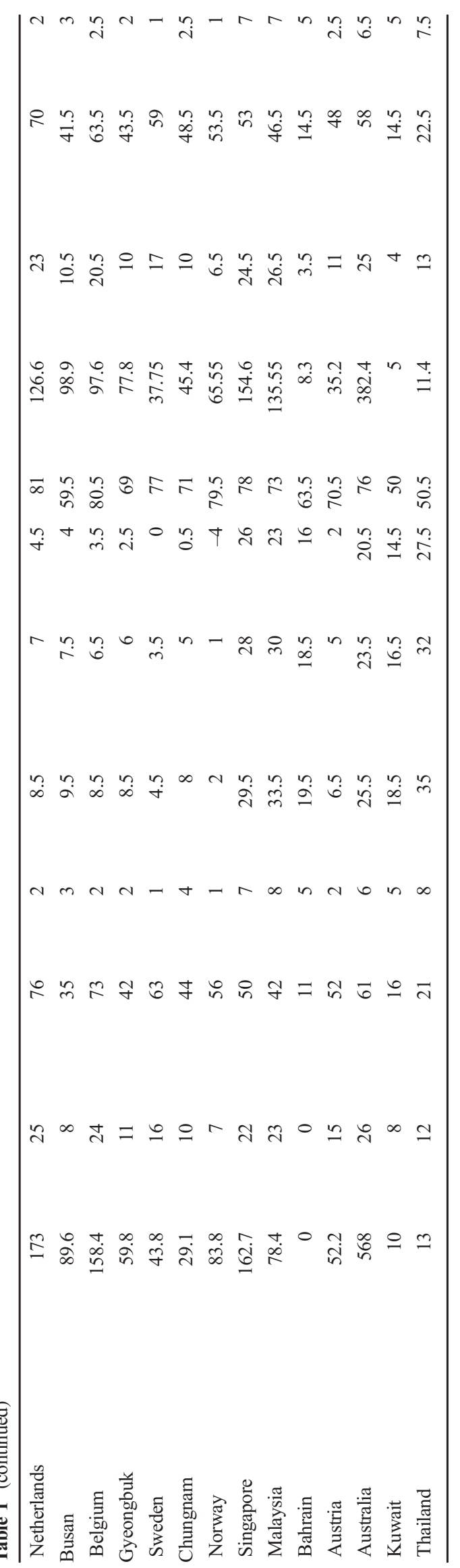




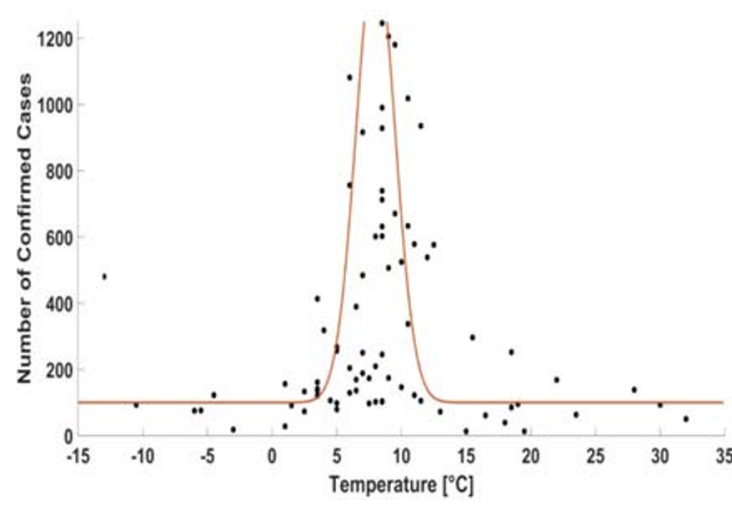

Fig. 1 Scatter plot of temperature and total number of confirmed cases up till 7th March 2020. Temperature data have been obtained from the online dataset as mentioned in the 'Materials and methods' section and were averaged over January and February 2020. a Scatter plot between average daily temperature and number of confirmed cases. The line

normal fit, with very narrow distribution. The point of Wuhan, where the maximum confirmed cases are reported, also lies within the peak (not shown in the figure). As evident from the figure, it clearly appears that the temperature range of $5-15^{\circ} \mathrm{C}$ comprises almost $90 \%$ of the total confirmed cases. With very small $\sigma$ value of the distribution, it is very apparent that the temperature band for community infection is, indeed, very narrow. Previous studies have clearly demonstrated that lower temperatures are directly related to influenza virus activity (Deyle et al. 2016; Ianevski et al. 2019; Roussel et al. 2016). Thus, it is clear that increasing temperature may have some clear indication for the reduction in the number of infected cases. Further, to understand if the diurnal variations in temperature, in addition to daily maximum temperature, has any impact on the infection among the community, we calculated the difference between the daily maximum and minimum temperatures and plotted it against the cumulative number of confirmed cases for respective temperature difference values (Fig. 1b). It is evident from the figure that the pronounced diurnal

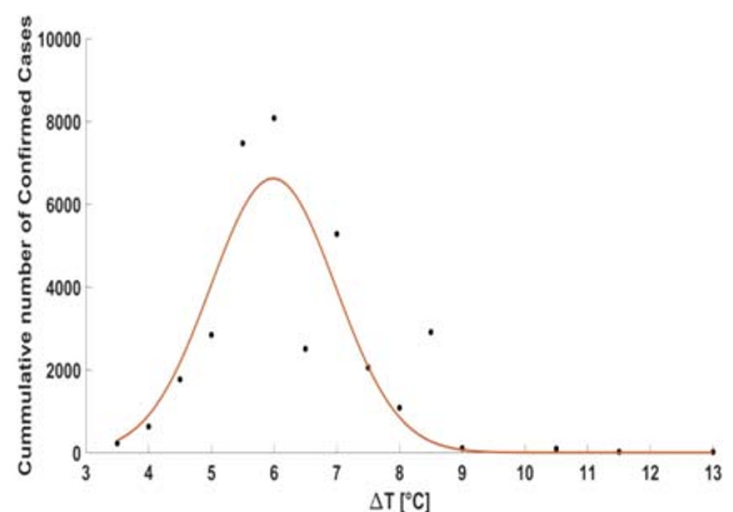

shows the log normal fit to the data $\left(N=85 ; R^{2}=0.35\right)$. b Scatter plot of the difference between the maximum and minimum daily temperatures and accumulated number of confirmed cases. The orange line shows the $\log$ normal fit to the data $\left(N=14 ; R^{2}=0.74\right)$. All the fits are statistically significant

variations with a difference of $\sim 7^{\circ} \mathrm{C}$ between the maximum and minimum temperatures have shown a consistent decrease in community infection. However, further investigations are required on if such a trend is also evident for warmer and relatively colder regions.

Further, interestingly, we also found a very strong relation between UV index and number of confirmed cases. As shown in Fig. 2, it is evident that the number cumulative cases were found to be highest for a UV index of 2.5 and gradually decreased from a UV index of 3.5. For the areas where the UV index was higher than 5 , the number of confirmed infected cases decreased further. It has been documented that higher temperature or prolonged exposure to UVC radiation lowers the virus infectivity (Ianevski et al. 2019). This is mainly resulting from the fact that increased temperatures and prolonged exposure to UV radiation are unable to kill the retinal pigment epithelium (RPE) layer of the cells. Warmer winters in Europe and the USA have been associated with reduced influenza virus activities (Ballester et al. 2016). As
Fig. 2 Scatter plot between cumulative confirmed cases and UV index (refer to the 'Materials and methods' section for more details). The daily UV index for the months of January and February was obtained from the source mentioned in the 'Materials and methods' section. The daily UV index data for the months of January and February were further averaged. The line indicates the spline fit to the data $\left(N=14 ; R^{2}=0.63\right)$

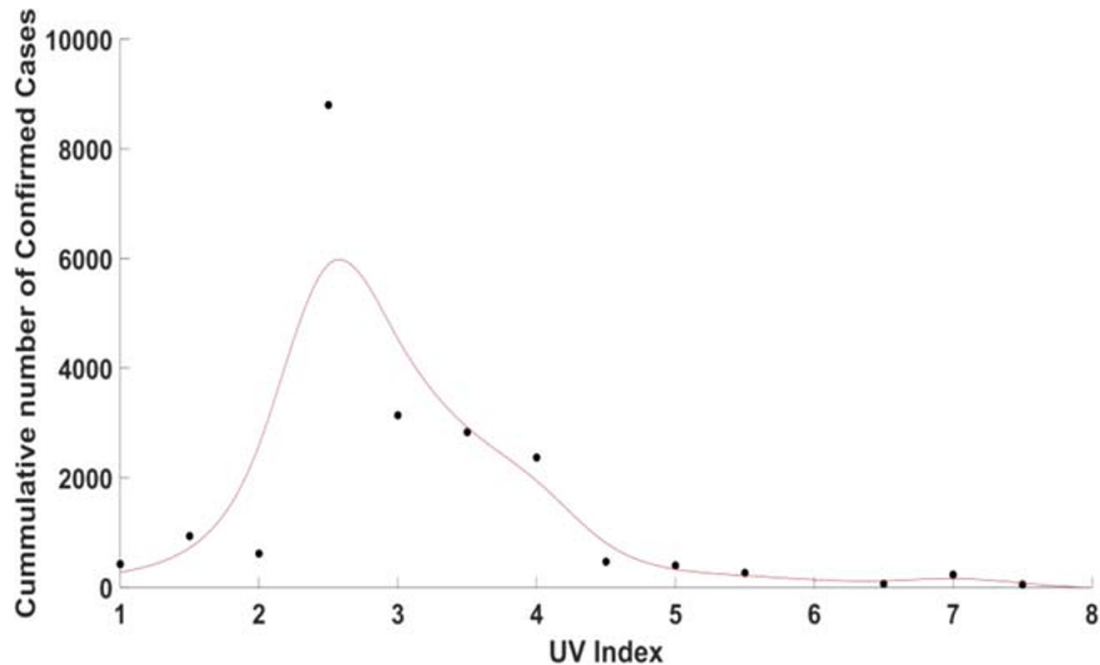




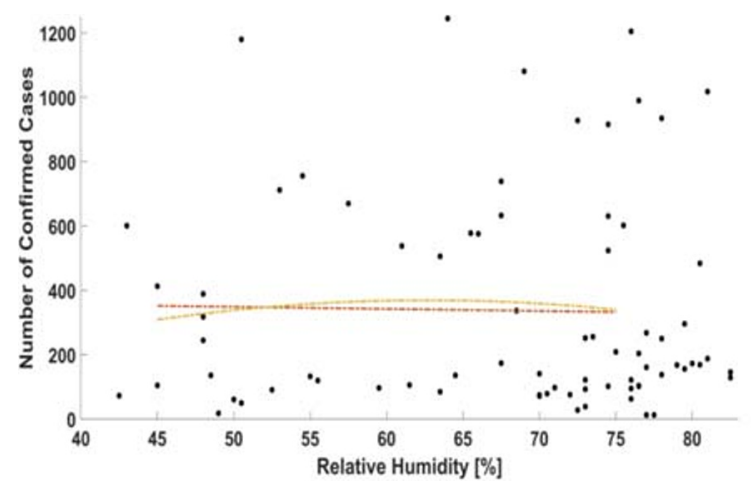

Fig. 3 Scatter plot between meteorological parameters and number of confirmed cases up till 7th March 2020. a Scatter plot between relative humidity $(\%)$ and number of confirmed cases, with the orange and red lines indicating squared and linear fit, respectively $\left(N=85 ; R^{2}=0.0005\right.$

shown in Fig. 3, we did not observe any strong dependence between the relative humidity and precipitation and community infection.

\section{Concluding remarks}

While the world is putting every possible effort into tackling the global epidemic situation resulting from the novel COVID-19 outbreak, very little information is available about the novel virus. Under this situation, in this study, we presented the possible dependence between the infection within a community and the temperature and UV index. By analyzing the data up to 7th March 2020 by considering more than 80 locations comprising of a number of confirmed cases and location-specific meteorological data, we showed that the spread of the virus is strongly dependent on the temperature and UV index. There is a very narrow temperature band of 3-

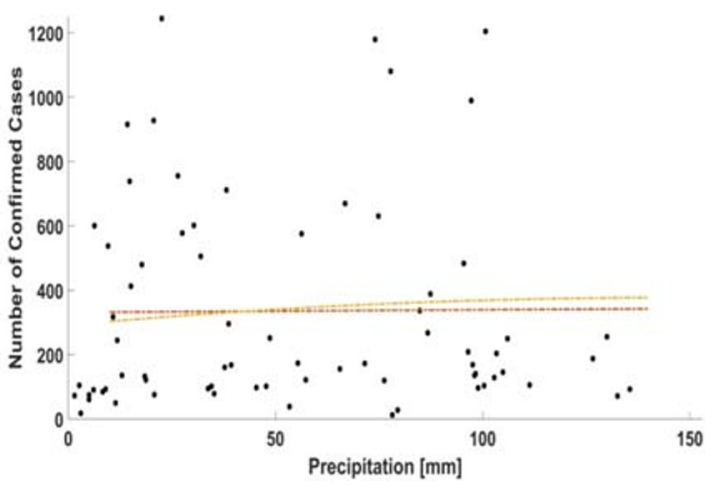

for linear fit and $R^{2}=0.007$ for squared fit). b Same as a but for precipitation $\left(N=85 ; R^{2}=0.0002\right.$ for linear fit and $R^{2}=0.01$ for squared fit

$12{ }^{\circ} \mathrm{C}$ where $90 \%$ of the total confirmed cases are agglomerated. As can be seen from Fig. 4, major outbreaks across the globe are located in the temperature areas lying in the range consistent with our analysis. Most importantly, increase of the UV index has also shown a decrease in the number of cases. This is evident from the fact that the global UV index map averaged over 5 days for representation purposes clearly shows a relatively lower UV index for the most affected areas (Fig. 5). This, in our opinion, is a very striking feature of our findings and we believe that artificial UV radiation could be one of the effective ways for sterilizing built-up environments for reducing spread amongst the community. While we believe that we are not presenting any physiological evidences for the decrease in community infections of COVID-19 due to increase in temperature and UV index, we strongly believe that this information could be very useful, particularly when pharmaceutical interventions are not possible at this moment to deal with the global crisis. It is important, however, to note
Fig. 4 Global map of the average temperature for January and February 2020, with the countries affected by more than 1000 cases as of 9 th March 2020 indicated by the black circles. Importantly, countries, regions and locations severely affected are in a very narrow band of latitude

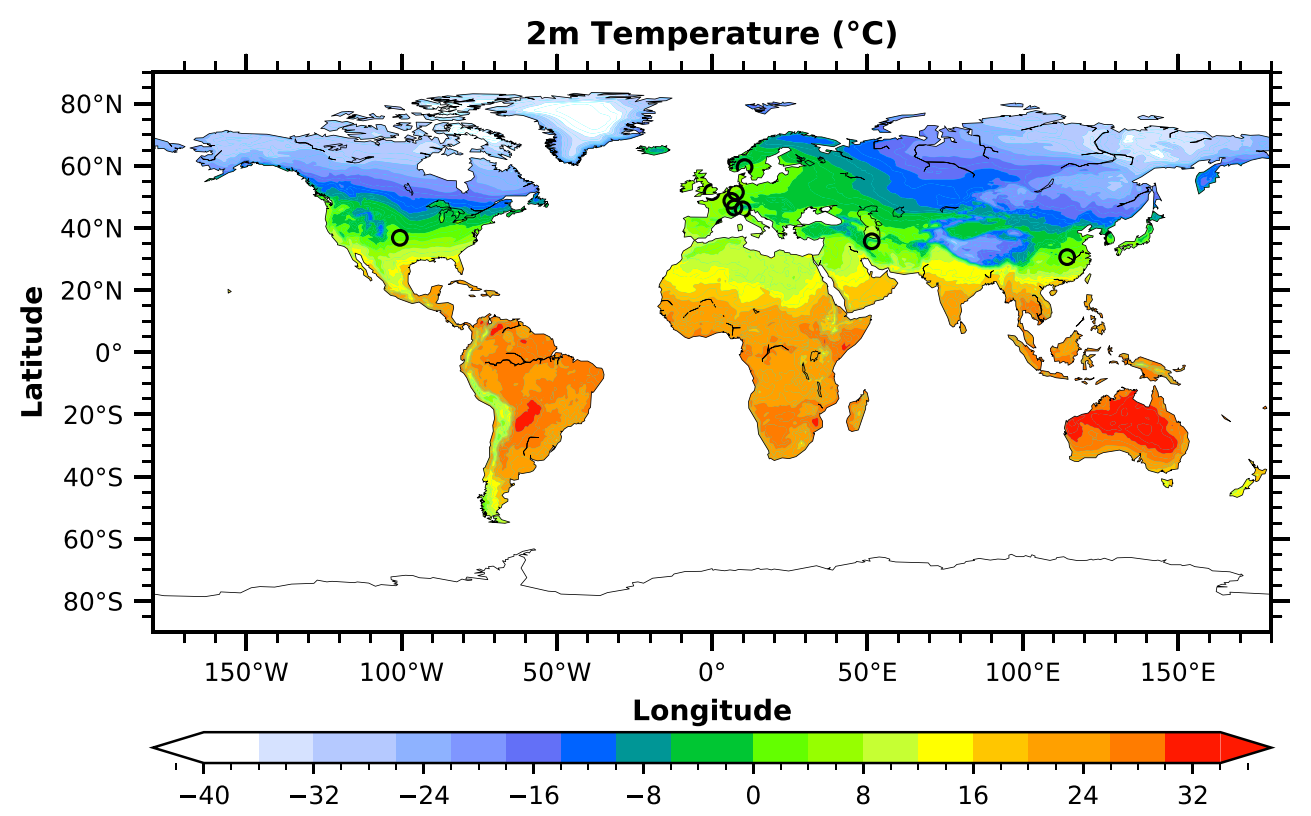


Fig. 5 Similar to Fig. 4 but representing the UV index and locations indicated by the dots. Again, a very narrow band of UV index is clearly evident for the locations that are severely affected

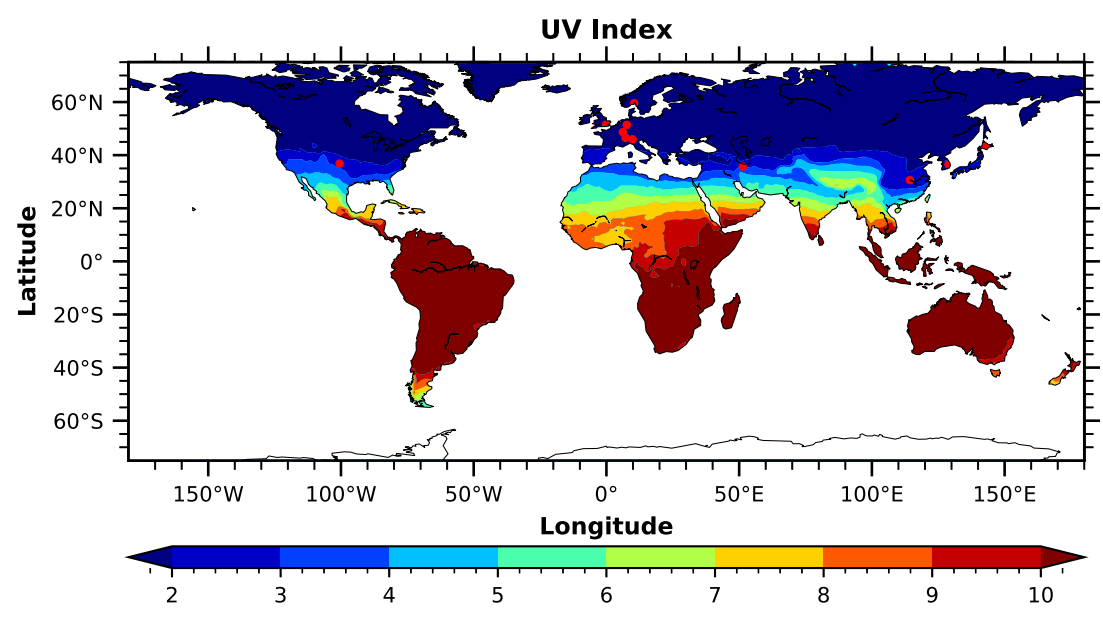

that the information provided here is very preliminary and is not based on any physiological studies involving either confirmed cases and/or viruses. We advise that preparedness and other measures should take this study only for information purposes and propose further detailed physiological studies.

Acknowledgements The authors duly acknowledge the Program for Monitoring Emerging Diseases (ProMED), the https://www. worldweatheronline.com service, and the UV global map used in this study was produced with the Giovanni online data system, developed and maintained by the NASA GES DISC. SSG acknowledges Nabha S. Gunthe and Nupura S. Gunthe for the encouragement and stimulating discussions during the preparation of this manuscript. SSG is thankful to selected faculty colleagues at Dept. of Civil Engineering at IIT Madras for helpful suggestions.

\section{Compliance with ethical standards Not applicable.}

Conflict of interest The authors declare that they do not have any conflict of interest.

Role of funding source Not applicable.

Ethical approval Not applicable.

\section{References}

Ballester J, Rodó X, Robine J-M, Herrmann FR (2016) European seasonal mortality and influenza incidence due to winter temperature variability. Nat Clim Change 6:927-930. https://doi.org/10.1038/ nclimate 3070

Chen PJ, Mao LJ, Nassis GP, Harmer P, AinsworthBE, Li F (2020) Coronavirus disease (COVID-19): the need to maintain regular physical activity while taking precautions. J Sport Health Sci 9: 103-104. https://doi.org/10.1016/j.jshs.2020.02.001
Deyle ER, Maher MC, Hernandez RD, Basu S, Sugihara G (2016) Global environmental drivers of influenza. Proc Natl Acad Sci USA 113: 13081-13086. https://doi.org/10.1073/pnas.1607747113

Du Toit A (2020) Outbreak of a novel coronavirus. Nat Rev Microbiol 18: 123. https://doi.org/10.1038/s41579-020-0332-0

Fisher MC, Henk DA, Briggs CJ et al (2012) Emerging fungal threats to animal, plant and ecosystem health. Nature 484:186-194. https:// doi.org/10.1038/nature10947

Fong SJ, Li G, Dey N, Crespo RG, Herrera-Viedma E (2020) Finding an accurate early forecasting model from small dataset: a case of 2019nCoV novel coronavirus outbreak. Int J Interact Multimed Artif Intell 6:132-140. https://doi.org/10.9781/ijimai.2020.02.002

Ianevski A, Zusinaite E, Shtaida N et al (2019) Low temperature and low UV indexes correlated with peaks of influenza virus activity in northern Europe during 2010-2018. Viruses 11:207. https://doi. org/10.3390/v11030207

Ibekwe PU, Ukonu BA (2019) Impact of weather conditions on atopic dermatitis prevalence in Abuja, Nigeria. J Natl Med Assoc 111:8893. https://doi.org/10.1016/j.jnma.2018.06.005

Ji W, Wang W, Zhao XF, Zai JJ, Li XG (2020) Cross-species transmission of the newly identified coronavirus 2019-nCoV. J Med Virol 92: 433-440. https://doi.org/10.1002/jmv.25682

Patel A, Jernigan DB; 2019-nCoV CDC Response Team (2020) Initial public health response and interim clinical guidance for the 2019 novel coronavirus outbreak - United States, December 31, 2019February 4, 2020 (reprinted from Recomm Rep, vol 68 2019). Am J Transplant 20:889-895. https://doi.org/10.1111/ajt.15805

Phan T (2020) Novel coronavirus: From discovery to clinical diagnostics. Infect Genet Evol 79:104211. https://doi.org/10.1016/j.meegid. 2020.104211

Roussel M, Pontier D, Cohen J-M, Lina B, Fouchet D (2016) Quantifying the role of weather on seasonal influenza. BMC Public Health 16: 441. https://doi.org/10.1186/s12889-016-3114-X

Yadav S, Gettu N, Swain B, Kumari K, Ojha N, Gunthe SS (2020) Bioaerosol impact on crop health over India due to emerging fungal diseases (EFDs): an important missing link. Environ Sci Pollut Res Int 27:12802-12829. https://doi.org/10.1007/s11356-020-08059-x

Publisher's note Springer Nature remains neutral with regard to jurisdictional claims in published maps and institutional affiliations. 